

\title{
Estimation of Lateral Dynamics and Road Curvature for Two-Wheeled Vehicles: A HOSM Observer approach
}

Mohammed El-Habib Dabladji, Dalil Ichalal, Hichem Arioui, Saïd Mammar

\section{To cite this version:}

Mohammed El-Habib Dabladji, Dalil Ichalal, Hichem Arioui, Saïd Mammar. Estimation of Lateral Dynamics and Road Curvature for Two-Wheeled Vehicles: A HOSM Observer approach. 19th IFAC World Congress on International Federation of Automatic Control (IFAC 2014), Aug 2014, Cape Town, South Africa. pp.2806-2811. hal-00964085

\section{HAL Id: hal-00964085 https://inria.hal.science/hal-00964085}

Submitted on 24 Mar 2014

HAL is a multi-disciplinary open access archive for the deposit and dissemination of scientific research documents, whether they are published or not. The documents may come from teaching and research institutions in France or abroad, or from public or private research centers.
L'archive ouverte pluridisciplinaire HAL, est destinée au dépôt et à la diffusion de documents scientifiques de niveau recherche, publiés ou non, émanant des établissements d'enseignement et de recherche français ou étrangers, des laboratoires publics ou privés. 


\title{
Estimation of Lateral Dynamics and Road Curvature for Two-Wheeled Vehicles: A HOSM Observer approach
}

\author{
Mohammed El-Habib Dabladji, Dalil Ichalal, \\ Hichem Arioui and Saïd Mammar* \\ * Informatique, Biologie Intégrative et Systèmes Complexes Laboratory \\ (IBISC EA-4526), Evry Val d'Essonne University (UEVE), 91020 \\ Evry, France, (e-mail: habib.dabladji@ibisc.univ-evry.fr).
}

\begin{abstract}
In this work, two high order sliding mode observers under cascade form are proposed in order to estimate the important states and inputs affecting the lateral dynamics of twowheeled vehicles. The first observer is based on a vision system and is used to estimate the lateral velocity of the vehicle. This velocity is considered as an additional measure for the second observer which is designed to estimate the tire forces, the roll angle and torque applied to the handle-bar.

The main contribution of this work is the estimation of the tire forces even with the variation of the longitudinal velocity and without the prior knowledge of the pneumatic coefficients.
\end{abstract}

Keywords: Two-Wheeled Vehicles, High Order Sliding Mode Observer, Vision System.

\section{INTRODUCTION}

Actually, the use of powered two-wheelers (PTW) is increasingly common thanks to the freedom of riding they offer and to the possibility to avoid congestion of the road. However, injuries of motorcyclists are out of proportion to their presence on roads. In the last French Technical report on Road Safety, the authors state that motorcyclists are just $2.5 \%$ of road traffic, but account for $26 \%$ of road user deaths (see ONISR (2012)).

In fact, nowadays, a growing delay is observed in the development of safety systems for motorcycles compared to other vehicles and the direct transportation of car safety systems to motorcycles is not obvious because of the complexity of the motorcycle dynamics and the influence of the rider on the behavior of the vehicle (see Evangelos (2010)). Moreover, motorcycle safety systems designers are faced with another problem, namely: the exact knowledge of the relevant dynamic states and inputs in order to quantify the risk (loss of control, etc.) or to catch a risk situation.

In this context, the motorcycle dynamics can be estimated through suitable sensors. However, this solution is not always obvious for several reasons: the price of some sensors (sensors of steering torque, etc.) or the feasibility of others (lateral tire forces sensors, etc.). On the other hand, sparsely works deal with the estimation of the PTW's dynamics.

In the literature, the estimation of the lateral dynamics has mainly concerned the lean ones with neglect of the steering mechanism. A frequency separation filtering observer (see Boniolo et al. (2009)) and extended Kalman filter (see Teerhuis and Jansen (2010)) were proposed to estimate the roll angle but with the neglect of the steering dynamics and by considering the tire-road forces in their linear form. In addition, these works are not robust to the variation of the forward velocity. The observation of the steering angle was proposed in De Filippi et al. (2011) with an LPV observer and scheduling gain technique missing the estimation convergence guarantee. More recently, a HOSM observer was proposed in Nehaoua et al. (2013) and a Takagi-Sugeno unknown input observer in Ichalal et al. (2013) to estimate the lateral dynamics and the steering torque. However, the lateral forces have been considered in their linear form and with known parameters. Moreover, for the former, it has been pointed that the observer was not robust to the variation of the longitudinal velocity which has been considered constant.

To the best of our knowledge, the estimation of the lean dynamics, the steering dynamics and the lateral tire forces in their nonlinear form, without the prior knowledge of their parameters and for a large range of forward velocities have never been addressed before for motorcycles.

\section{PROBLEM STATEMENT}

Our long term objective is to assist the rider in a passive or an active way in order to anticipate or to remedy a dangerous situations. Thus, we must identify all the pertinent parameters that affect the dynamics of PTW vehicles. The exact knowledge of such parameters is important to improve the risk quantification of the loss-of-control during cornering (see Slimi et al. (2010)) for example or the stoppie phenomenon during hard braking (see Corno et al. (2008)).

It is well known in the motorcycle literature that the tire is one of the motorcycle's most important components (see Cossalter (2006)). In addition to the comfort of the ride that the tire procures, it improves the adherence which is 
necessary to the generation of the longitudinal forces for acceleration and braking and also to the lateral forces for corning movements.

In this context, it seems necessary to estimate either the adherence or the tire forces. In our case, we are interesting by the cornering situations and we try to estimate the lateral forces in their nonlinear form even when the pneumatic parameters are unknown or change with time. Indeed and in the proposed work, we deal with the UIHOSMO (Unknown Input High Order Sliding Mode Observers) (see Fridman et al. (2007)) for dynamics state observation and unknown inputs reconstruction. To be more consistent with realistic constraints, we consider the tire forces as unknown inputs to avoid the prior knowledge of their parameters.

Of course, Sliding Mode Observers (SMO) has been successfully developed in the variable structure theory (see Edwards et al. (2002)). SMO are attractive because of their insensitivity w.r.t unknown inputs and the possibility of using equivalent output injection to obtain additional information and to reconstruct the unknown inputs (seeFridman et al. (2011)). More recently, and based on super twisting algorithms (see Levant (1993)), robust exact differentiator has been proved to be a powerful tool to estimate in finite time the derivative of signals (see Levant (1998)). Under the condition of minimum phase, and by an adequate system coordinate transformation, an UIHOSMO based on the robust exact differentiator will be applied in order to estimate all the unknown states and inputs.

The presented paper is organized as follows: in section 2 , we give a brief theoretical background on the observability and detectability of linear parameter varying systems.. In section 3 , the vision system and the motorcycle lateral dynamics models are described. The cascaded HOSMO is described in section 4 . The simulation results of this work are given in section 5.The paper is wrap up by some conclusions.

\section{THEORETICAL BACKGROUND}

In this paper, we deal with the following class of systems:

$$
\begin{aligned}
& \dot{x}=A(\theta) x+D(\theta) d \\
& y=C x
\end{aligned}
$$

where $x \in \mathcal{R}^{n}$ is the state vector, $d \in \mathcal{R}^{m}$ is the unknown input vector, $y \in \mathcal{R}^{m}$ is the measured output vector and $\theta \in \Sigma \subset \mathcal{R}^{q}$ is an external measured vector.

Firstly, we recall some definitions and propositions about observability and detectability. We consider also that the vector $\theta=\theta_{0}$ is piecewise constant with: $A(\theta)=A^{*}$ and $D(\theta)=D^{*}$

Definition 1. For the system (1), we define the Roosenbrock matrix of the triplet $\left(A^{*}, D^{*}, C\right)$ as:

$$
R(s)=\left[\begin{array}{cc}
s I-A^{*} & D^{*} \\
-C & 0
\end{array}\right]
$$

$s_{0}$ is called an invariant zero of the triplet $\left(A^{*}, D^{*}, C\right)$ if $\operatorname{rank} R\left(s_{0}\right)<n+\operatorname{rank} D$.
Definition 2. Consider the system (1) with $\theta=\theta_{0}$. $\left(r_{1}, \ldots, r_{p}\right)$ is called the vector of partial relative degrees of the output vector $y$ w.r.t the unknown input vector, if for each partial relative degree $r_{i}$, the following equations hold:

$$
\begin{aligned}
C_{i} A^{* j} D^{*} & =0, j=1, \ldots, r_{i}-2 \\
C_{i} A^{* r_{i}-1} D^{*} & \neq 0
\end{aligned}
$$

and

$$
\operatorname{det}\left[\begin{array}{c}
C_{1} A^{* r_{1}-1} D^{*} \\
\vdots \\
C_{p} A^{* r_{p}-1} D^{*}
\end{array}\right] \neq 0
$$

where $C_{i}$ is the $i^{\text {th }}$ line vector of the matrix $C$.

We call $r=\Sigma_{1}^{p} r_{i}$ the relative degree of the output vector $y$ w.r.t the unknown input $d$. It is always less than or equal to $n$.

Definition 3. The system (1) is called strongly observable if:

$$
\left[\forall x(0) \in \mathcal{R}^{n}, \forall d(t) \in \mathcal{R}^{m}, y(t) \equiv 0\right] \Rightarrow[x(t) \equiv 0]
$$

Otherwise, it is called strongly detectable if:

$\left[\forall x(0) \in \mathcal{R}^{n}, \forall d(t) \in \mathcal{R}^{m}, y(t) \equiv 0\right] \Rightarrow[x(t) \rightarrow 0$ as $t \rightarrow 0](6)$

Proposition 4. The system (1) with $\theta=\theta_{0}$ is called strongly observable if and only if one of these statements holds:

- The triplet $\left(A^{*}, D^{*}, C\right)$ has no invariant zeros.

- The output vector $y$ has a relative degree $r=n$ w.r.t the unknown input $d$.

Otherwise, it is called strongly detectable if and only if:

- The relative degree $r$ of the output vector $y$ w.r.t the unknown input vector $d$ exists and the triplet $\left(A^{*}, D^{*}, C\right)$ has no unstable invariant zeros. (i.e. The system is minimum phase).

Now and to design our observers, we must describe the vision system and the motorcycle lateral dynamics models.

\section{MOTORCYCLE MODEL DESCRIPTION}

In this section, we describe the model of the vision system xbased on the lateral displacement (figure ??) and the motorcycle lateral dynamics based on the model of Sharp 1971 with 2 body frames and 4 degrees of freedom (DOF) (figure 2).

The main aim of adding a vision system to our structure is to estimate the lateral velocity which will give us an additional degree of freedom in the reconstruction of the lateral forces. This is described more in details in section 5 .

An overall scheme of the system structure linked to the observer is in figure 1.

\subsection{Vision system model}

We consider using a vision system giving us the lateral displacement of the vehicle from the centerline at a look 


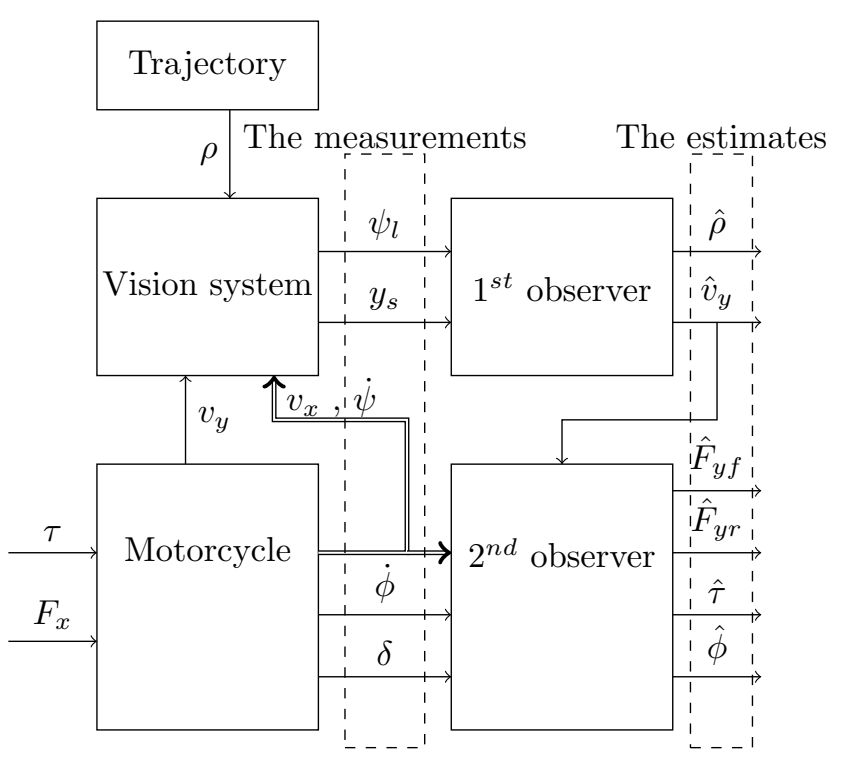

Fig. 1. Overall scheme of the motorcycle with the vision system and the observer

ahead distance and the angular displacement (figure ??). These measures are extracted from images obtained with a suitable camera.

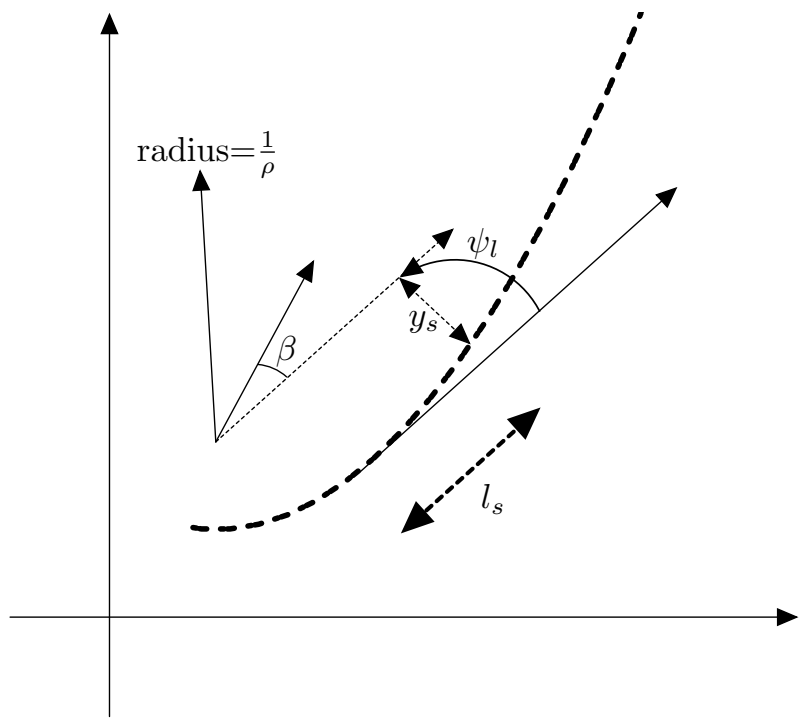

The equations describing the evolution of theses measurements are given by:

$$
\begin{aligned}
& \dot{y}_{s}=v_{x}\left(\beta+\psi_{l}\right)+l_{s}\left(\dot{\psi}-v_{x} \rho\right) \\
& \dot{\psi}_{l}=\dot{\psi}-v_{x} \rho
\end{aligned}
$$

where $y_{s}$ is the offset from the centerline at a look ahead distance $l_{s}, \psi_{l}$ is the angular displacement, $\beta$ is the lateral slip angle $\left(\beta=\tan ^{-1}\left(\frac{v_{y}-b \dot{\psi}}{v_{x}}\right)\right), v_{y}$ is the lateral velocity, $v_{x}$ is the longitudinal velocity, $\dot{\psi}$ is the yaw rate of the vehicle and $\rho$ is the road curvature.

The system (7) can be rewritten in the form:

$$
\begin{aligned}
\dot{x}_{1} & =\left(\begin{array}{cc}
0 & v_{x} \\
0 & 0
\end{array}\right) x_{1}+\left(\begin{array}{cc}
v_{x} & -l_{s} v_{x} \\
0 & -v_{x}
\end{array}\right) d_{1}+\left(\begin{array}{c}
l_{s} \\
1
\end{array}\right) \dot{\psi} \\
& =A_{1} x_{1}+D_{1} d_{1}+E_{1} y_{1}
\end{aligned}
$$

$$
y_{1}=C_{1} x_{1}
$$

where $x_{1}^{T}=\left(y_{s} \psi_{l}\right), d_{1}^{T}=(\beta \rho)$ and $C_{1}=\left[\begin{array}{ll}1 & 0 \\ 0 & 1\end{array}\right]$.

The vision system's parameters are described with their numerical values in appendix A.

Remark 5. Note that the model (7) used for the vision system is available only for small roll angles. Of course, for high roll angles, the model of the vision system will be coupled with the lean dynamics.

In our context, we are interested by urban riding situations and we consider the hypothesis of small roll angle available.

Discussion on the observability For the model (7) and for $v_{x}=v_{x}^{*}$, The Roosenbrock matrix is given by:

$$
R_{1}(s)=\left[\begin{array}{cccc}
s & -v_{x}^{*} & v_{x}^{*} & -l_{s} v_{x}^{*} \\
0 & s & 0 & -v_{x}^{*} \\
1 & 0 & 0 & 0 \\
0 & 1 & 0 & 0
\end{array}\right]
$$

If $v_{x}^{*} \neq 0$, then the Roosenbrock matrix is always full rank. So, the system (7) is always strongly observable for $v_{x}^{*} \neq 0$.

\subsection{Motorcycle lateral dynamics}

In this work, we consider only the lateral dynamics. Our work is based on the model of Sharp (1971). The motorcycle is represented as two linked bodies: the front and the main frame. The former includes the handlebar assembly and the front wheel, where the former contains the chassis, the engine and the rear wheel.

Thus, the obtained model is considered having 4 DOF: the yaw dynamics, the roll dynamics, the lateral displacement of the main frame and the rotation of the steering system (the front frame) (figure 2).

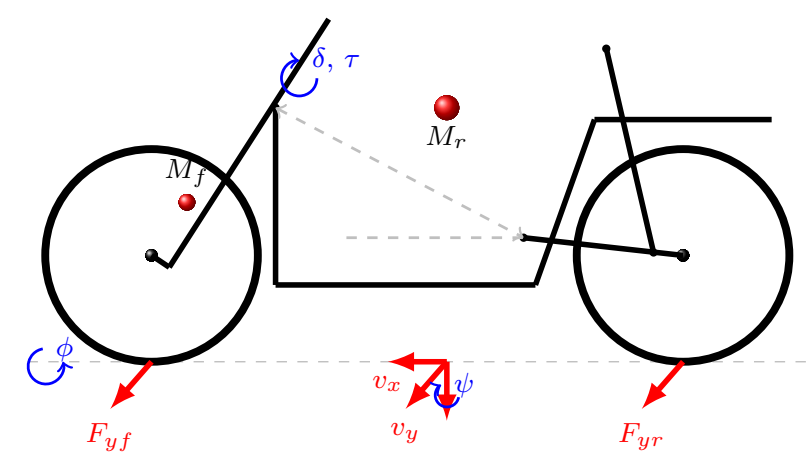

Fig. 2. Geometrical representation of the Sharp's motorcycle model

Instead of considering the tire forces in a linear form as reported in Sharp (1971), they are expressed in this work using the magic formula of Pacejka (2005). Moreover, we consider that the tire characteristics are unknown which is more realistic because the tire characteristics change with the tire's pressure and the road's adherence.

Thereby, we obtain the following differential equations: 


$$
\begin{cases}M\left(\dot{v}_{y}+v_{x} \dot{\psi}\right)+M_{f} k \ddot{\psi}+d_{1} \ddot{\phi}+M_{f} e \ddot{\delta} & =F_{y f}+F_{y r} \\ M_{f} k\left(\dot{v}_{y}+v_{x} \dot{\psi}\right)+a_{2} \ddot{\phi}+a_{3} \ddot{\psi}+a_{1} \ddot{\delta}-a_{4} v_{x} \dot{\phi}-d_{2} v_{x} \dot{\delta} & =\sum M_{z} \\ d_{1} \dot{v}_{y}+b_{2} \ddot{\phi}+a_{2} \ddot{\psi}+b_{1} \ddot{\delta}+b_{5} v_{x} \dot{\psi}+d_{3} v_{x} \dot{\delta} & =\sum M_{x} \\ M_{f} e \dot{v}_{y}+b_{1} \ddot{\phi}+a_{1} \ddot{\psi}+c_{1} \ddot{\delta}-d_{3} v_{x} \dot{\phi}+c_{3} v_{x} \dot{\psi}+K \dot{\delta} & =\sum M_{s}\end{cases}
$$

where:

$$
\begin{aligned}
\sum M_{z} & =l_{f} F_{y f}-l_{r} F_{y r} \\
\sum M_{x} & =b_{4} \sin (\phi)-b_{3} \sin (\delta) \\
\sum M_{s} & =-b_{3} \sin (\phi)-c_{2} \sin (\delta)-\eta F_{y f}+\tau \\
F_{y f} & =D_{f} \cdot \sin \left\{C_{1 f} \cdot \arctan \left[B_{1 f} \alpha_{f}-E_{1 f}\left(B_{1 f} \alpha_{f}-\arctan B_{1 f} \alpha_{f}\right)\right]\right. \\
& \left.+C_{2 f} \cdot \arctan \left[B_{2 f} \theta_{f}-E_{2 f}\left(B_{2 f} \theta_{f}-\arctan B_{2 f} \theta_{f}\right)\right]\right\} \\
F_{y r} & =D_{r} \cdot \sin \left\{C_{1 r} \cdot \arctan \left[B_{1 r} \alpha_{r}-E_{1 r}\left(B_{1 r} \alpha_{r}-\arctan B_{1 r} \alpha_{r}\right)\right]\right. \\
& \left.+C_{2 r} \cdot \arctan \left[B_{2 r} \theta_{r}-E_{2 r}\left(B_{2 r} \theta_{r}-\arctan B_{2 r} \theta_{r}\right)\right]\right\}
\end{aligned}
$$

$\alpha_{f}=\tan ^{-1}\left[\left(\frac{v_{y}+l_{f} \dot{\psi}-\eta \dot{\delta}}{v_{x}}\right)-\delta \cos (\varepsilon)\right]$ and $\alpha_{r}=\beta$ are the front and rear side slip angles and $\theta_{f}=\phi+\delta \sin (\varepsilon)$ and $\theta_{r}=\phi$ the front and rear camber angles respectively.

$M_{f}, M_{r}$ and $M$ are the mass of the front, the main frame and whole the vehicle respectively, $\phi$ is the roll angle, $\delta$ is the steering angle, $F_{y f}$ and $F_{y r}$ are the lateral front and rear forces respectively and $\tau$ is the torque applied to the handle bar. More details on the motorcycle parameters and expressions are given in appendix $\mathrm{A}$.

Instead of including the tire forces in the dynamic state representation, they are considered as unknown inputs. Note that the torque applied to the handle-bar is hard to measure. To this fact, it is also considered as an unknown input.

With this configuration, we will have a state representation with 6 states $\left(\phi, \delta, v_{y}, \dot{\psi}, \dot{\phi}, \dot{\delta}\right)$ and 3 unknown inputs $\left(F_{y f}, F_{y r}, \tau\right)$. The measurement are roll rate $\dot{\phi}$, the yaw rate $\dot{\psi}$ and the steering angle $\delta$.

However, it is well known in the bicycle and motorcycle literature that the lateral dynamics are modeled by a nonminimum phase system when the steering dynamics are taking into account (the counter steering phenomena). With the latter state representation, we will obtain an unstable zero. Therefore, the system is neither strongly observable nor strongly detectable.

In order to make the system strongly observable, we consider the roll angle as an unknown input (see Nehaoua et al. (2013)). Thus, we obtain the following state representation:

$$
\begin{gathered}
\left(\begin{array}{ccccc}
1 & 0 & 0 & 0 & 0 \\
0 & M_{f} e & M & M_{f} k & d_{1} \\
0 & a_{1} & M_{f} k & a_{3} & a_{2} \\
0 & b_{1} & d_{1} & a_{2} & b_{2} \\
0 & c_{1} & M_{f} e & a_{1} & b_{1}
\end{array}\right) \dot{x}_{2}= \\
\left(\begin{array}{ccccc}
0 & 1 & 0 & 0 & 0 \\
0 & 0 & 0 & -M v_{x} & 0 \\
0 & d_{2} v_{x} & 0 & -M_{f} k v_{x} & a_{4} v_{x} \\
-b_{3} & d_{3} v_{x} & 0 & b_{5} v_{x} & 0 \\
-c_{2} & -K & 0 & -c_{3} v_{x} & d_{1} v_{x}
\end{array}\right) x_{2}+\left(\begin{array}{ccccc}
0 & 0 & 0 & 0 & 0 \\
1 & 1 & 0 & 0 \\
l_{f} & -l_{r} & 0 & 0 \\
0 & 0 & 0 & b_{4} \\
-\eta & 0 & 1 & -b_{3}
\end{array}\right) d_{2}
\end{gathered}
$$

(10)

The dimension of the vector of unknown inputs is 4 . So, we need to at least 4 measures to be able to reconstruct the unknown inputs; which motivates the addition of the vision system to obtain the lateral velocity $v_{y}$ as the fourth measure for our observer. Thus, the output vector become:

$$
\begin{aligned}
y_{2} & =\left[\begin{array}{lllll}
1 & 0 & 0 & 0 & 0 \\
0 & 0 & 1 & 0 & 0 \\
0 & 0 & 0 & 1 & 0 \\
0 & 0 & 0 & 0 & 1
\end{array}\right] x_{2} \\
& =C_{2} x_{2}
\end{aligned}
$$

Discussion on the observability For the motorcycle model (12) and for a longitudinal velocity $v_{x}=v_{x}^{*}$, the Roosenbrock matrix is given by:

$$
R_{2}(s)=\left[\begin{array}{cc}
s I_{4}-M^{-1} A_{2}^{*} & M^{-1} D_{2} \\
C_{2} & 0
\end{array}\right]
$$

The matrix $R_{2}(s)$ is full rank if and only if $R_{2}(s)$ given by (15) is full rank.

$$
\dot{R}_{2}(s)=\left[\begin{array}{cc}
s M-A_{2}^{*} & D_{2} \\
C_{2} & 0
\end{array}\right]
$$

After computation, we find: $\operatorname{det}\left(\dot{R}_{2}(s)\right)=b_{4}\left(l_{f}+l_{r}\right)$. Knowing that $l_{f}$ and $l_{r}$ are geometrical parameters and are always greater than 0 , also $b_{4}=\left(M_{f} j+M_{r} h\right) g \neq 0$. So, $\operatorname{det}\left(\hat{R}_{2}(s)\right)$ is always full rank and the system (12) is strongly observable.

The determinant of $\dot{R}_{2}(s)$ is $b_{4}\left(l_{f}+l_{r}\right)$ which is always positive. Knowing that lf and $\mathrm{lr}$ are geometrical parameters and are always greater than 0 , also b4 $=(\mathrm{Mf} \mathrm{j}+\mathrm{Mrh}) \mathrm{g}$ $6=0$. So, D2 is always full rank. So, $R_{2}(s)$ has always full rank, regardless of the longitudinal velocity. Thus, the system (12) is strongly observable.

\section{OBSERVER DESIGN}

Now, we will design a cascaded observer in order to estimate the tire forces, the lateral velocity, the roll angle, the road curvature and the steering torque. The diagram of the proposed observer is given in figure (1).

\subsection{Observer design for the vision system}

The first observer is based on the vision system which is modeled by the system (7). We consider that all the state vector $x_{1}$ is measured. Indeed, the partial relative degrees vector is: $\left(r_{1}, r_{2}\right)=(1,1)$ and as seen before, the system is strongly observable.

Note that the system (7) is already written in the triangular form w.r.t each output. Moreover, all the states are measured. So, we do not need the transformation suggested in other papers (see Fridman et al. (2011)) to reconstruct the unknown input vector. Moreover, since we do not need this transformation, the proposed observer is insensitive to longitudinal velocity variations. 
Since the two outputs $y_{s}$ and $\psi_{l}$ and their firsts derivatives are bounded, we use a second order sliding mode differentiator to estimate the first derivative of the output vector.

$$
\begin{aligned}
& \dot{v}_{11}=v_{12}-\lambda_{11}\left|v_{11}-y_{s}\right|^{\frac{1}{2}} \operatorname{sign}\left(v_{11}-y_{s}\right) \\
& \dot{v}_{12}=-\lambda_{12} \operatorname{sign}\left(v_{12}-\dot{v}_{11}\right) \\
& \dot{v}_{21}=v_{22}-\lambda_{21}\left|v_{21}-\psi_{l}\right|^{\frac{1}{2}} \operatorname{sign}\left(v_{21}-\psi_{l}\right) \\
& \dot{v}_{22}=-\lambda_{22} \operatorname{sign}\left(v_{22}-\dot{v}_{21}\right)
\end{aligned}
$$

Let $v_{1}$ and $v_{2}$ the estimates of $x_{1}$ and its derivative $\dot{x}_{1}$. We have: $v_{1}^{T}=\left(v_{11}, v_{21}\right)$ and $v_{2}^{T}=\left(v_{12}, v_{22}\right)$.

From (7) and the expressions of the estimates and thanks to the fact that $D_{1}$ is full rank, the estimate of the unknown input vector is given by:

$$
\hat{d}_{1}=D_{1}^{-1}\left[v_{2}-A_{1} v_{1}-E_{1} y_{1}\right]
$$

with $d_{1}^{T}=[\hat{\beta}, \hat{\rho}]$.

Remark 6 . The only condition for the existence of the unknown input observer is that: the forward velocity $v_{x}$ must not be null (i.e. the vehicle must not be at rest) to garantee the full rank of the matrice $D_{1}$.

Now, from $\hat{\beta}=\tan ^{-1}\left(\frac{\hat{v}_{y}-b \dot{\psi}}{v_{x}}\right)$, we obtain:

$$
\hat{v}_{y}=v_{x} \tan \hat{\beta}+b \dot{\psi}
$$

\subsection{Observer design for the motorcycle lateral dynamics}

For the second observer, we consider the lateral velocity as an additional measure in order to make the number of measures equal to the number on the unknown inputs. This hypothesis is available thanks to the finite time convergence of the first observer.

In this case, we see that the partial relative degree of the output vector w.r.t the unknown input is $(2,1,1$, 1) independently of the longitudinal velocity. Thus, the system is strongly observable and the states and unknown inputs can be estimated accurately.

As well as for the first observer, we will use high order differentiators in order to estimate $\dot{\delta}, \ddot{\delta}, \dot{v}_{y}, \ddot{\phi}, \ddot{\psi}$.

Thus, we propose the following differentiator:

$$
\begin{aligned}
& \dot{v}_{31}=v_{32}-\lambda_{31}\left|v_{31}-\delta\right|^{\frac{2}{3}} \operatorname{sign}\left(v_{31}-\delta\right) \\
& \dot{v}_{32}=v_{33}-\lambda_{32}\left|v_{32}-\dot{v}_{31}\right|^{\frac{1}{2}} \operatorname{sign}\left(v_{32}-\dot{v}_{31}\right) \\
& \dot{v}_{33}=-\lambda_{33} \operatorname{sign}\left(v_{33}-\dot{v}_{32}\right) \\
& \dot{v}_{41}=v_{42}-\lambda_{41}\left|v_{41}-\hat{v}_{y}\right|^{\frac{1}{2}} \operatorname{sign}\left(v_{41}-\hat{v}_{y}\right) \\
& \dot{v}_{42}=-\lambda_{42} \operatorname{sign}\left(v_{42}-\dot{v}_{41}\right) \\
& \dot{v}_{51}=v_{52}-\lambda_{51}\left|v_{51}-\dot{\psi}\right|^{\frac{1}{2}} \operatorname{sign}\left(v_{51}-\dot{\psi}\right) \\
& \dot{v}_{52}=-\lambda_{52} \operatorname{sign}\left(v_{52}-\dot{v}_{51}\right) \\
& \dot{v}_{61}=v_{62}-\lambda_{61}\left|v_{61}-\dot{\phi}\right|^{\frac{1}{2}} \operatorname{sign}\left(v_{61}-\dot{\phi}\right) \\
& \dot{v}_{62}=-\lambda_{62} \operatorname{sign}\left(v_{62}-\dot{v}_{61}\right)
\end{aligned}
$$

Thus the estimate of $x_{2}$ and $\dot{x}_{2}$ are given by:

$$
\xi_{1}^{T}=\left(v_{31}, v_{32}, v_{41}, v_{51}, v_{61}\right)
$$

$$
\xi_{2}^{T}=\left(v_{32}, v_{33}, v_{42}, v_{52}, v_{62}\right)
$$

Finally, the estimate of $d_{2}$ is obtained by combining (20) with (12):

$$
d_{2}=D_{2}^{-1}\left[M_{2} \xi_{2}-A_{2} \xi_{1}\right]
$$

Thus, we have estimated the lateral forces, the steering torque, the roll angle and the steering rate.

Remark 7. The condition to the existence of such an observer is the full rank of the matrix $D_{2}$. Of course, $D_{2}$ is full rank because $b_{4} \neq 0$ and $l_{f}+l_{r} \neq 0$.

\section{SIMULATION RESULTS}

The simulations are carried out on a non-linear model including the longitudinal and the lateral dynamics. The tire forces are modeled by the magic formula of Pacejka. The simulation conditions are given for a lane change maneuver and for the longitudinal velocity profile given in figure (3).

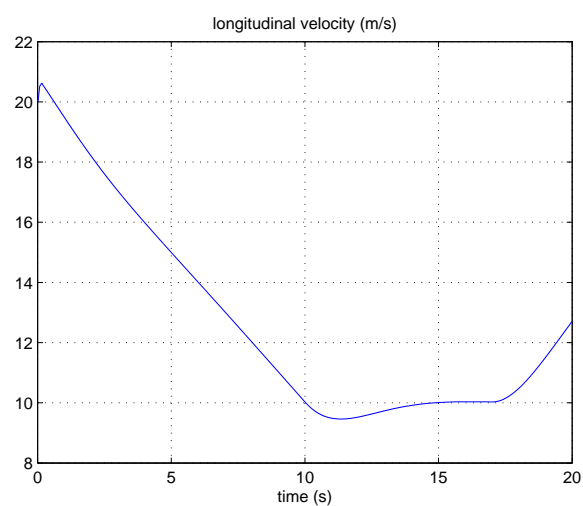

Fig. 3. Longitudinal velocity profile

The results of simulation without noises for the first observer are given in figure (4) and for the second observer in figure(5).
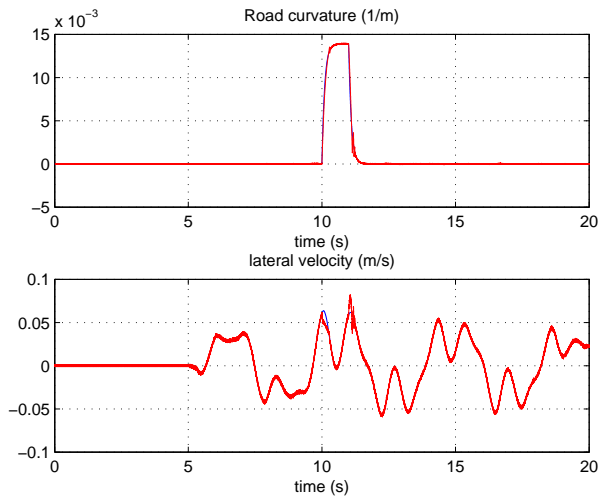

Fig. 4. (top) The road curvature (blue) and its estimated (red), (below) the lateral velocity (blue) and its estimated (red)

Without noises, we see that all the parameters are well estimated. The main advantage of this observer is that we need any knowledge of the pneumatic parameters. Only 

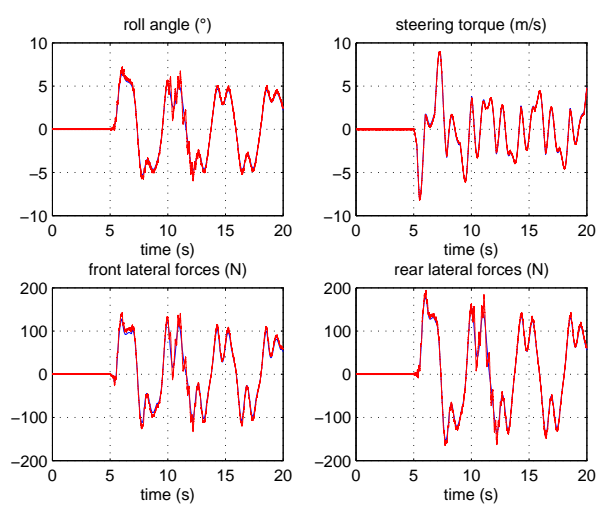

Fig. 5. (top) The unknown inputs (blue) and their estimates (red)

the geometric parameters and the mass of the motorcycle are required.

Another advantage of this observer is its robustness to the variation on the longitudinal velocity, which has been considered variable in simulation.

Now, we consider that the measures are affected by a centered and random noises in order to test the observer in the presence of noised measurements. The simulation results are depicted in figure (6) and (7). The effect of noises is visible, but the estimation of all the parameters is acceptable.

Of course, we have a compromise in the chose of the differentiator gains. If they are chosen sufficiently large, we will have good and fast estimation, but the observer will be very sensitive to noises. And in the other case, the observer will be less sensitive to noises but the estimation of the unknown signals will not be accurate.

Also, it should be noted that with this kind of differentiator, the obtained estimates are sensitive to the sampling period. In this context, several works have been proposed recently to remedy to this problem (see Ibrir (2003)).


Fig. 6. (top) The road curvature (blue) and its estimated (red), (below) the lateral velocity (blue) and its estimated (red): the noisy case


Fig. 7. (top) The unknown inputs (blue) and their estimates (red): : the noisy case

\section{CONCLUSION}

In this work, an UIHOSMO is proposed for the PTW vehicles to estimate the lateral dynamics, the tire forces and the road curvature. The vehicle is equipped with a vision system in order to estimate firstly the lateral velocity and the road curvature using HOSM differentiator. Next we consider the estimated lateral velocity as an auxiliary output for the second system thanks to the finite time convergence of differentiator. Based on the same technique of derivation, and on the second system, the tire forces, the roll angle and the steering torque - which are all considered as unknown inputs - are estimated in finite time also. It is pointed out that the proposed observer is insensitive to variations of the longitudinal velocity and needs any prior knowledge of the pneumatic parameters. Only the measures given by sensors, the masses and the geometrical parameters are needed. To our knowledge, such a work has never been addressed before for motorcycles. Validation results on an experimental scooter will be published in future works.

\section{REFERENCES}

Boniolo, I., Savaresi, S., and Tanelli, M. (2009). Roll angle estimation in two-wheeled vehicles. Control Theory Applications, IET, 3(1), 20-32.

Corno, M., Savaresi, S.M., Tanelli, M., and Fabbri, L. (2008). On optimal motorcycle braking. Control Engineering Practice, 16, $644-657$.

Cossalter, V. (2006). Motorcycle Dynamics.

De Filippi, P., Corno, M., Tanelli, M., and Savaresi, S. (2011). Single-sensor control strategies for semiactive steering damper control in two-wheeled vehicles. Vehicular Technology, IEEE Transactions on, 61(2), 813-820. doi:10.1109/TVT.2011.2180031.

Edwards, C., Spurgeon, S., and Tan, C. (2002). On the development and application of sliding mode observers. In Variable Structure Systems: Towards the 21st Century. Springer Berlin Heidelberg.

Evangelos, B. (2010). Saferider project. Technical report, French National Agency of Reaserch.

Fridman, L., Davila, J., and Levant, A. (2011). Highorder sliding-mode observation for linear systems with unknown inputs. Nonlinear Analysis: Hybrid Systems, $5(2), 189-205$. 
Fridman, L., Levant, A., and Davila, J. (2007). Observation of linear systems with unknown inputs via highorder sliding-modes. International Journal of Systems Science, 38, 773-791.

Ibrir, S. (2003). Online exact differentiation and notion of asymptotic algebraic observers. Automatic Control, IEEE Transactions on, 48(11), 2055-2060. doi: 10.1109/TAC.2003.819303.

Ichalal, D., Arioui, H., and Mammar, S. (2013). Observer design for motorcycle lean and steering dynamics estimation: a takagi-sugeno approach. In American Control Conference (ACC).

Levant, A. (1993). Sliding order and sliding accuracy in sliding mode control. International Journal of Control, $58,1247-1263$.

Levant, A. (1998). Robust exact differentiation via sliding mode technique. Automatica, 34, 379 - 384.

Nehaoua, L., Ichalal, D., Arioui, H., Mammar, S., and Fridman, L. (2013). Lean and steering motorcycle dynamics reconstruction : An unknown input HOSMO approach. In American Control Conference (ACC).

ONISR (2012). Characteristics and causes of road accidents 2011. Technical report, French Interministerial National Observatory of Road Safety. URL http://www . securite-routiere.gouv.fr.

Pacejka, H. (2005). Tire and Vehicle Dynamics. SAE International.

Sharp, R.S. (1971). The stability and control of motorcycles. Mechanical Engineering Science, 13, 316 - 329.

Slimi, H., Arioui, H., Nouveliere, L., and Mammar, S. (2010). Motorcycle speed profile in cornering situation. In American Control Conference ( $A C C)$.

Teerhuis, A.P. and Jansen, S.T. (2010). Motorcycle state estimation for lateral dynamics. In Bicycle and Motorcycle Dynamics.

\section{Appendix A. SYSTEM'S PARAMETERS} DESCRIPTION AND NUMERICAL VALUES

Table A.1. Vision system and motorcycle dynamic variables

\begin{tabular}{|c|c|}
\hline \multicolumn{2}{|l|}{ Vision system } \\
\hline$y_{s}, \psi_{l}$ & $\begin{array}{l}\text { offset and angular displacement at a look } \\
\text { ahead distance }\end{array}$ \\
\hline$l_{s}$ & look ahead distance \\
\hline$\rho$ & road curvature \\
\hline$\beta$ & lateral slip angle \\
\hline \multicolumn{2}{|l|}{ Motorcycle } \\
\hline$v_{x}, v_{y}$ & longitudinal and lateral velocities \\
\hline$\phi, \psi, \delta$ & roll, yaw and steering angles \\
\hline$F_{y f}, F_{y r}$ & front and rear lateral forces \\
\hline$\tau$ & steering torque \\
\hline$M_{f}, M_{r}, M$ & $\begin{array}{l}\text { mass of the front frame, the rear frame } \\
\text { and the whole motorcycle }\end{array}$ \\
\hline$K$ & $\begin{array}{l}\text { damper coefficient of the steering } \\
\text { mechanism }\end{array}$ \\
\hline$B_{i j}, C_{i j}, D_{j}, E_{i j}$ & $\begin{array}{l}\text { Magic formula pneumatic parameters } \\
i \in(1,2), j \in(f, r)\end{array}$ \\
\hline
\end{tabular}

The other parameters $a_{i}, b_{i}, c_{i}$ and $d_{i}$ are described in table A.2
Table A.2. Motorcycle parameters expressions and numerical values

\begin{tabular}{l}
\hline parameters $a_{i}, b_{i}, c_{i}$ and $d_{i}$ \\
\hline$a_{1}=M_{f} e k+I_{f z} \cos \epsilon$ \\
$a_{2}=M_{f} j k-C_{r x z}+\left(I_{f z}-I_{f x}\right) \sin \epsilon \cos \epsilon$ \\
$a_{3}=M_{f} k^{2}+I_{r z}+I_{f x} \sin ^{2} \epsilon+I_{f z} \cos ^{2} \epsilon, a_{4}=\frac{i_{f y}}{R_{f}}+\frac{i_{r y}}{R_{r}}$ \\
$b_{1}=M_{f} e j+I_{f z} \sin \epsilon$ \\
$b_{2}=M_{f} j^{2}+M_{r} h^{2}+I_{r x}+I_{f x} \cos ^{2} \epsilon+I_{f z} \sin ^{2} \epsilon$ \\
$b_{3}=\eta Z_{f}-M_{f} e g, b_{4}=\left(M_{f} j+M_{r} h\right) g$ \\
$b_{5}=M_{f} j+M_{r} h+\frac{i_{f y}}{R_{f}}+\frac{i_{r y}}{R_{r}}, c_{1}=I_{f z}+M_{f} e^{2}$ \\
$c_{2}=\left(\eta Z_{f}-M_{f} e g\right) \sin \epsilon, c_{3}=M_{f} e+\frac{i_{f y}}{R_{f}} \sin \epsilon$ \\
$d_{1}=M_{f} j+M_{r} h, d_{2}=\frac{i_{f y}}{R_{f}} \sin \epsilon, d_{3}=-\frac{i_{f y}}{R_{f}} \cos \epsilon$ \\
\hline Parameters $a_{i}, b_{i}, c_{i}$ and $d_{i} \operatorname{contain}$ the geometrical \\
parameters of the motorcycle and their signification are \\
illustrated in Sharp $(1971)$ \\
\hline Numerical values \\
\hline$l_{s}=1 m, M_{f}=30.65 k g, M_{r}=217.45 k g, M=M_{f}+M_{r}$ \\
$K=6.77 N . s / r a d, I_{r x}=31.18 k g / m^{2}, I_{r z}=21.07 k g / m^{2}$ \\
$g=9.81 m / s^{2}, C_{r x z}=1.74 k g / m^{2}, I_{f x}=1.24 k g / m^{2}$ \\
$I_{f z}=0.44 k g / m m^{2}, i_{f y}=0.72 k g / m^{2}, i_{r y}=1.05 k g / m^{2}$ \\
$a=0.949 m, e=0.024 m, f=0.028 m, h=0.616 m$ \\
$R_{f}=0.305 m, R_{r}=0.305 m, \eta=0.116 m, \epsilon=0.47^{\circ}$ \\
$k=(a+e) \cos \epsilon-f \sin \epsilon, j=(a+e) \sin \epsilon+f \cos \epsilon$ \\
$l_{r}=0.585 m, l_{f}=0.829 m, e m p=l f+l r, Z_{f}=-\frac{M}{e m p} l_{r} g$
\end{tabular}

